\title{
Isothiazoloquinolones with Enhanced
}

\section{Antistaphylococcal Activities against Multi-Drug-}

\section{Resistant Strains: Effects of Structural Modifications at}

\section{the 6-, 7-, and 8-Positions}

Qiuping Wang, Edlaine Lucien, Akihiro Hashimoto, Godwin C. G. Pais, David M. Nelson, Yongsheng

Song, Jane A. Thanassi, Christopher W. Marlor, Christy L. Thoma, Jijun Cheng, Steven D. Podos, Yangsi Ou, Milind Deshpande, Michael J. Pucci, Douglas D. Buechter, Barton J. Bradbury, and Jason A. Wiles*

Achillion Pharmaceuticals, Inc., 300 George Street, New Haven, Connecticut 06511

* To whom correspondence should be addressed. Telephone: 203-624-7000. Fax: 203-752-5454. Email: jwiles@achillion.com. 
Table S1. HPLC Purity Data for Target Compounds

\begin{tabular}{|c|c|c|c|c|}
\hline \multirow{2}{*}{ Compd } & \multicolumn{2}{|c|}{ Method $1^{a}$} & \multicolumn{2}{|c|}{ Method $2^{b}$} \\
\hline & $\overline{t_{\mathrm{R}}(\min )}$ & Purity (\%) & $t_{\mathrm{R}}(\min )$ & Purity (\%) \\
\hline $5 \mathbf{a}$ & 8.33 & 95.1 & 12.29 & 99.3 \\
\hline $5 b$ & 8.70 & 99.3 & 12.88 & 97.1 \\
\hline $5 c$ & 8.49 & 95.8 & 12.39 & 97.1 \\
\hline $5 e$ & 8.30 & 97.2 & 12.59 & 96.9 \\
\hline $5 f$ & 6.80 & 99.1 & 10.27 & 99.5 \\
\hline $5 g$ & 7.28 & 99.4 & 10.67 & 99.7 \\
\hline $5 h$ & 7.41 & 96.5 & 10.77 & 96.7 \\
\hline 6f & 7.07 & 99.2 & 10.88 & 99.7 \\
\hline $6 g$ & 7.20 & 95.9 & 10.84 & 98.2 \\
\hline $6 h$ & 7.45 & 96.1 & 11.41 & 95.2 \\
\hline $7 \mathbf{a}$ & 8.51 & 99.7 & 12.25 & 99.9 \\
\hline $7 \mathbf{b}$ & 8.84 & 99.3 & 13.08 & 98.7 \\
\hline $7 c$ & 8.71 & 97.2 & 12.83 & 98.6 \\
\hline $7 d$ & 8.75 & 99.2 & 12.55 & 98.7 \\
\hline $7 e$ & 8.90 & 99.9 & 12.37 & 92.3 \\
\hline $7 f$ & 7.34 & 98.9 & 10.84 & 99.9 \\
\hline $7 g$ & 7.58 & 99.5 & 10.82 & 99.8 \\
\hline $7 \mathrm{~h}$ & 7.82 & 98.7 & 13.08 & 98.7 \\
\hline $8 \mathbf{a}$ & 8.42 & 99.5 & 12.29 & 96.0 \\
\hline $8 b$ & 8.75 & 97.9 & 12.88 & 95.6 \\
\hline $8 c$ & 8.63 & 99.3 & 12.52 & 98.0 \\
\hline $8 d$ & 9.25 & 99.0 & 12.20 & 99.5 \\
\hline $8 e$ & 8.51 & 95.7 & 12.20 & 96.3 \\
\hline $8 f$ & 7.12 & 99.4 & 10.08 & 99.4 \\
\hline $8 g$ & 7.46 & 99.2 & 10.64 & 99.4 \\
\hline $8 h$ & 7.75 & 98.7 & 11.07 & 99.4 \\
\hline
\end{tabular}

${ }^{a}$ HPLC conditions: 20-min gradient elution of increasing concentrations of $\mathrm{CH}_{3} \mathrm{CN}$ in water (5-95\%) containing $0.1 \%$ TFA with a flow rate of $1.0 \mathrm{~mL} / \mathrm{min}$ and UV detection at $254 \mathrm{~nm}$ on a Waters X-bridge C18 $150 \times 4.6 \mathrm{~mm} 3.5 \mu \mathrm{m}$ column.

${ }^{b}$ HPLC conditions: 20-min gradient elution of increasing concentrations of $\mathrm{MeOH}$ in water (5-95\%) containing $0.1 \%$ TFA with a flow rate of $1.0 \mathrm{~mL} / \mathrm{min}$ and $\mathrm{UV}$ detection at $254 \mathrm{~nm}$ on a Waters X-bridge C8 $150 \times 4.6 \mathrm{~mm} 3.5 \mu \mathrm{m}$ column. 
Table S2. Combustion Analyses Data for Target Compounds

\begin{tabular}{|c|c|c|c|c|c|}
\hline Compd & Formula & & $\mathrm{C}(\%)$ & $\mathrm{H}(\%)$ & $\mathrm{N}(\%)$ \\
\hline \multirow[t]{2}{*}{ 5f } & \multirow{2}{*}{$\mathrm{C}_{18} \mathrm{H}_{12} \mathrm{FN}_{3} \mathrm{O}_{2} \mathrm{~S} \cdot \mathrm{HCl} \cdot \mathrm{H}_{2} \mathrm{O}$} & Calcd & 53.01 & 3.71 & 10.30 \\
\hline & & Found & 53.16 & 3.46 & 9.99 \\
\hline \multirow[t]{2}{*}{$5 g$} & \multirow{2}{*}{$\mathrm{C}_{19} \mathrm{H}_{14} \mathrm{FN}_{3} \mathrm{O}_{2} \mathrm{~S} \cdot \mathrm{HCl} \cdot 1.8 \mathrm{H}_{2} \mathrm{O}$} & Calcd & 52.31 & 4.30 & 9.63 \\
\hline & & Found & 52.09 & 4.04 & 9.55 \\
\hline \multirow[t]{2}{*}{$6 f$} & \multirow[t]{2}{*}{$\mathrm{C}_{17} \mathrm{H}_{11} \mathrm{FN}_{4} \mathrm{O}_{2} \mathrm{~S} \cdot \mathrm{HCl} \cdot 0.4 \mathrm{H}_{2} \mathrm{O}$} & Calcd & 51.30 & 3.24 & 14.08 \\
\hline & & Found & 51.26 & 3.08 & 13.90 \\
\hline \multirow[t]{2}{*}{$6 \mathrm{~g}$} & \multirow[t]{2}{*}{$\mathrm{C}_{18} \mathrm{H}_{13} \mathrm{FN}_{4} \mathrm{O}_{2} \mathrm{~S} \cdot \mathrm{HCl} \cdot 1.3 \mathrm{H}_{2} \mathrm{O}$} & Calcd & 50.48 & 3.91 & 13.08 \\
\hline & & Found & 50.55 & 3.87 & 12.89 \\
\hline \multirow[t]{2}{*}{$7 \mathbf{a}$} & \multirow[t]{2}{*}{$\mathrm{C}_{22} \mathrm{H}_{18} \mathrm{FN}_{3} \mathrm{O}_{3} \mathrm{~S} \cdot \mathrm{HCl} \cdot 2.0 \mathrm{H}_{2} \mathrm{O}$} & Calcd & 53.28 & 4.67 & 8.47 \\
\hline & & Found & 53.62 & 4.30 & 8.09 \\
\hline \multirow[t]{2}{*}{$7 \mathbf{b}$} & \multirow{2}{*}{$\mathrm{C}_{23} \mathrm{H}_{20} \mathrm{FN}_{3} \mathrm{O}_{3} \mathrm{~S} \cdot \mathrm{HCl} \cdot 2.0 \mathrm{H}_{2} \mathrm{O}$} & Calcd & 54.17 & 4.94 & 8.24 \\
\hline & & Found & 54.08 & 4.68 & 8.17 \\
\hline \multirow[t]{2}{*}{$7 f$} & \multirow{2}{*}{$\mathrm{C}_{19} \mathrm{H}_{14} \mathrm{FN}_{3} \mathrm{O}_{3} \mathrm{~S} \cdot \mathrm{HCl} \cdot 1.2 \mathrm{H}_{2} \mathrm{O}$} & Calcd & 51.69 & 3.97 & 9.52 \\
\hline & & Found & 51.68 & 3.80 & 9.36 \\
\hline \multirow[t]{2}{*}{$7 \mathrm{~g}$} & \multirow[t]{2}{*}{$\mathrm{C}_{20} \mathrm{H}_{16} \mathrm{FN}_{3} \mathrm{O}_{3} \mathrm{~S} \cdot \mathrm{HCl} \cdot 1.1 \mathrm{H}_{2} \mathrm{O}$} & Calcd & 52.95 & 4.27 & 9.26 \\
\hline & & Found & 52.87 & 4.12 & 9.18 \\
\hline \multirow[t]{2}{*}{$7 \mathbf{h}$} & \multirow{2}{*}{$\mathrm{C}_{21} \mathrm{H}_{18} \mathrm{FN}_{3} \mathrm{O}_{3} \mathrm{~S} \cdot \mathrm{HCl} \cdot 1.8 \mathrm{H}_{2} \mathrm{O}$} & Calcd & 52.51 & 4.74 & 8.75 \\
\hline & & Found & 52.22 & 4.39 & 8.67 \\
\hline \multirow[t]{2}{*}{$8 f$} & \multirow[t]{2}{*}{$\mathrm{C}_{19} \mathrm{H}_{15} \mathrm{~N}_{3} \mathrm{O}_{3} \mathrm{~S} \cdot \mathrm{HCl} \cdot 1.5 \mathrm{H}_{2} \mathrm{O}$} & Calcd & 53.21 & 4.47 & 9.80 \\
\hline & & Found & 53.32 & 4.13 & 9.77 \\
\hline \multirow[t]{2}{*}{$8 \mathbf{h}$} & \multirow[t]{2}{*}{$\mathrm{C}_{21} \mathrm{H}_{19} \mathrm{~N}_{3} \mathrm{O}_{3} \mathrm{~S} \cdot \mathrm{HCl} \cdot 2.5 \mathrm{H}_{2} \mathrm{O}$} & Calcd & 53.11 & 5.31 & 8.85 \\
\hline & & Found & 53.16 & 5.05 & 8.79 \\
\hline
\end{tabular}

